\title{
Lung-sparing intensity-modulated radiotherapy in malignant pleural mesothelioma: palliative or potentially radical?
}

\author{
Angela Botticella ${ }^{1,2}$, Dirk De Ruysscher ${ }^{1,3}$ \\ ${ }^{1}$ KU Leuven - University of Leuven, Department of Oncology, Laboratory of Experimental Radiotherapy, Leuven, Belgium; ${ }^{2}$ Gustave Roussy Cancer \\ Campus, Department of Radiation Oncology, Villejuif, France; ${ }^{3}$ Department of Radiation Oncology (MAASTRO clinic), Maastricht University \\ Medical Centre, GROW, Mastricht, The Netherlands \\ Correspondence to: Angela Botticella, MD. Department of Radiation Oncology, Gustave Roussy Cancer Campus, 114 rue Edouard Vaillant, 94800 \\ Villejuif, France. Email: angela.botticella@gustaveroussy.fr. \\ Provenance: This is an invited Editorial commissioned by the Section Editor Laura Chiara Guglielmetti (Cantonal Hospital Winterthur, Kantonsspital \\ Winterthur, Winterthur, Switzerland). \\ Comment on: Parisi E, Romeo A, Sarnelli A, et al. High dose irradiation after pleurectomy/decortication or biopsy for pleural mesothelioma treatment. \\ Cancer Radiother 2017;21:766-73.
}

Submitted Sep 06, 2018. Accepted for publication Sep 11, 2018.

doi: $10.21037 /$ jtd.2018.09.47

View this article at: http://dx.doi.org/10.21037/jtd.2018.09.47

Malignant pleural mesothelioma (MPM) is a rare and aggressive tumour and its prognosis is dismal. The ASCO and the ESMO guidelines both place a platinumpemetrexed (or raltitrexed)-based chemotherapy for 4 to 6 cycles as standard of care in fit patients $(1,2)$. However, the median overall survival (OS) is only approximately 12 months, with hardly any 5 -year survivors (2).

In recent years, the role of surgery and the role of radiotherapy have been both redefined. New surgical techniques (less invasive compared to the extrapleural pneumonectomy-EPP), such as the lung-sparing pleurectomy/decortication (P/D), have been increasingly used, mainly due to the high post-operative mortality and morbidity of EPP, even in specialized centres (3).

Recent advances in radiotherapy planning and delivery have enabled dose escalation while maintaining acceptable dose constraints to normal tissues, even after a lungsparing surgery, thus in patients with two intact lungs. The MSKCC group has been the leader in optimizing lungsparing intensity-modulated radiotherapy (IMRT) (4-6), with encouraging results (median OS from the end of radiotherapy of 20.2 months (6). The median delivered dose was 46.8 Gy and $90 \%$ of patient received chemotherapy. Toxicity was acceptable, with a grade $\geq 3$ radiation pneumonitis rate between $13.6 \%$ and $20 \%(5,7)$. However, up to now, few groups confirmed the MSKCC data on lung-sparing IMRT $(8,9)$.
The report of Parisi and colleagues is therefore of great interest. They reported the results of a retrospective study in biopsy-proven MPM patients, in which 25 Gy in 5 fractions (or 37.5 Gy in 5 fractions, if residual disease after P/D) were delivered to the hemi-pleura. This palliative lungsparing IMRT was preceded by chemotherapy and/or lungsparing surgery $(\mathrm{P} / \mathrm{D})$.

The main objective was to investigate the feasibility of such a regimen in a cohort of patients with intact lungs (after biopsy or P/D). Thirty-six patients were retrospectively included: 19/36 (52.8\%) underwent P/D, $80 \%$ received chemotherapy and $14 / 36$ (39\%) were stage III (N2 mediastinal lymph nodes).

The median OS was 21.6 months. The proposed treatment was well tolerated, with $8 \%$ of patients who experienced grade $\geq 3$ radiation pneumonitis, $17 \%$ grade 2 cough and $14 \%$ grade 2 dyspnea.

There are several limitations to this study. First of all, this is not a clinical trial but a retrospective analysis of a group of patients treated with a nonstandard of care protocol. This raises serious concerns as to whether the patients truly gave informed consent to be entered in this protocol and what alternative options were offered to them. The latter is particularly relevant for the $41 \%$ of patients who presented with stage I-II disease and received chemotherapy and a palliative radiotherapy). One of the main limitations of MPM literature is the lack of high-quality data, and we 
strongly believe that, whenever possible, those patients should be included in prospective clinical trials.

Second, the objective of this analysis is to evaluate the feasibility of a palliative regimen in MPM patients after biopsy or P/D in terms of toxicity. However, little is said about the palliation of the symptoms (dyspnoea or thoracic), i.e., did this palliative IMRT reduce symptoms?

Nevertheless, the reported OS is nearly 22 months, which compares favourably with published prospective studies in patients who received higher doses of radiotherapy.

The results may therefore raise the hypothesis that lungsparing IMRT may lead to a promising OS in selected patients, but that high doses may not be necessary for achieving this.

It is hoped that the authors and centers will continue to pursue this strategy in a prospective study, and integrate this with chemotherapy and new drugs such as immune therapy (10).

\section{Acknowledgements}

Funding: $\mathrm{AB}$ was supported by a grant from the Stichting tegen kanker/Fondation contre le cancer (CA/2014/354) and by Kom op Tegen Kanker (Stand up to Cancer, The Flemish Cancer Society).

\section{Footnote}

Conflicts of Interest: The authors have no conflicts of interest to declare.

\section{References}

1. Baas P, Fennell D, Kerr KM, et al. Malignant pleural mesothelioma: ESMO Clinical Practice Guidelines for diagnosis, treatment and follow-up. Ann Oncol 2015;26 Suppl 5:v31-9.

2. Kindler HL, Ismaila N, Armato SG 3rd, et al. Treatment of Malignant Pleural Mesothelioma: American Society

Cite this article as: Botticella A, De Ruysscher D. Lungsparing intensity-modulated radiotherapy in malignant pleural mesothelioma: palliative or potentially radical? J Thorac Dis 2018;10(Suppl 33):S4038-S4039. doi: 10.21037/jtd.2018.09.47 of Clinical Oncology Clinical Practice Guideline. J Clin Oncol 2018;36:1343-73.

3. Sugarbaker DJ, Richards WG, Bueno R. Extrapleural pneumonectomy in the treatment of epithelioid malignant pleural mesothelioma: novel prognostic implications of combined N1 and N2 nodal involvement based on experience in 529 patients. Ann Surg 2014;260:577-80; discussion 580-2.

4. Rimner A, Zauderer MG, Gomez DR, et al. Phase II Study of Hemithoracic Intensity-Modulated Pleural Radiation Therapy (IMPRINT) As Part of Lung-Sparing Multimodality Therapy in Patients With Malignant Pleural Mesothelioma. J Clin Oncol 2016;34:2761-8.

5. Rosenzweig KE, Zauderer MG, Laser B, et al. Pleural intensity-modulated radiotherapy for malignant pleural mesothelioma. Int J Radiat Oncol Biol Phys 2012;83:1278-83.

6. Shaikh F, Zauderer MG, von Reibnitz D, et al. Improved Outcomes with Modern Lung-Sparing Trimodality Therapy in Patients with Malignant Pleural Mesothelioma. J Thorac Oncol 2017;12:993-1000.

7. Yorke ED, Jackson A, Kuo LC, et al. Heart Dosimetry is Correlated With Risk of Radiation Pneumonitis After Lung-Sparing Hemithoracic Pleural Intensity Modulated Radiation Therapy for Malignant Pleural Mesothelioma. Int J Radiat Oncol Biol Phys 2017;99:61-69.

8. Minatel E, Trovo M, Bearz A, et al. Radical Radiation Therapy After Lung-Sparing Surgery for Malignant Pleural Mesothelioma: Survival, Pattern of Failure, and Prognostic Factors. Int J Radiat Oncol Biol Phys 2015;93:606-13.

9. Minatel E, Trovo M, Polesel J, et al. Radical pleurectomy/ decortication followed by high dose of radiation therapy for malignant pleural mesothelioma. Final results with long-term follow-up. Lung Cancer 2014;83:78-82.

10. Quispel-Janssen J, van der Noort V, de Vries JF, et al. Programmed Death 1 Blockade With Nivolumab in Patients With Recurrent Malignant Pleural Mesothelioma. J Thorac Oncol 2018. [Epub ahead of print]. 